\title{
Atrial Closure Device
}

National Cancer Institute

\section{Source}

National Cancer Institute. Atrial Closure Device. NCI Thesaurus. Code C80466.

A wire mesh device used for the closure of atrial septal defects. 